\title{
Optimal Auctions in Oligopoly Spectrum Market with Concealed Cost
}

\author{
Raouf Abozariba \\ School of Comp. and Communications \\ Lancaster University \\ Lancaster, UK \\ r.abozariba@ieee.org
}

\author{
Md Asaduzzaman \\ School of Creative Arts and Eng. \\ Staffordshire University \\ Stoke on Trent, UK \\ asad@ieee.org
}

\author{
Mohammad N. Patwary \\ School of Comp. and Digital Tech. \\ Birmingham City University \\ Birmingham, UK \\ mohammad.patwary@bcu.ac.uk
}

\begin{abstract}
This paper presents a mathematical approach to the future dynamic spectrum market, where multiple secondary operators compete to gain radio resources. The secondary network operators (SNOs) face various concurrent auctions. We discuss techniques, which can be used to select auctions to optimize their objectives and increase the winning probability. To achieve these goals, a matching problem is formulated and solved, where secondary operators are paired with auctions, which can provide spectrum with the highest expected quality of service $(\mathrm{QoS})$. A total outlay optimization is structured for auctions with concealed reserve prices, which are only revealed to the secondary operators for some price upon request. More specifically, we solve a nonlinear problem to determine the minimum set of auctions by using the brute force algorithm. We further introduce a surplus maximization and demonstrate an auction mechanism of spectrum allocation by modifying the Bayesian-Nash equilibrium. The mathematical analyses highlight that the optimal choice is achievable through the proposed mathematical formulation.

Index Terms-Auction mechanism, Hierarchical optimization problem, Optimal spectrum purchasing.
\end{abstract}

\section{INTRODUCTION}

Uses of wireless communication continue to be introduced in areas such as augmented and virtual reality, industrial control, automated driving or flying, robotics, and tactile internet [1], [2]. Such applications and their requirements will demand large supply of radio resources to facilitate communications [3], [4]. With the current distribution of licensed spectrum bandwidths combined with the scarcity of unlicensed spectrum, operators will seek a rental approach to provide consistent spectrum resources to connect users' equipments [5]. It is forecast that by 2020 there will be more than 50 billion connected devices and the majority of them will use cellular networks for communications [6]. Clearly, to serve such a large number of terminals, future networks will have to dramatically increase the provided capacity compared to present standards [7]. However, spectrum resources remain under the ownership of governmental agencies and major industry players and licenses to use the spectrum require large capital outlays and are granted for many years making it harder for new emerging technologies to develop at the desired rate. Using current approaches to achieve the ambitious goal of providing connectivity to billions of devices is therefore not sustainable. The launch of the fifth-generation (5G) of mobile cellular networks, with millions more base stations, is envisaged to support these terminals with their applications and diverse requirements in terms of latency and reliability [8].

Dynamic spectrum allocation facilitates access to $5 \mathrm{G}$ and its supported data network. However, spectrum allocation is a well-studied area and can be performed broadly two ways: pre-purchased [9]-[12] and auction based [13]-[15]. Among auction based techniques to trade spectrum between operators, Gandhi et al. propose a real-time spectrum auction framework [16]. Using auctions allows the spectrum owners to ask for bids from potential secondary operators, to allocate spectrum resources and to charge payments as a function of the received bids [17]-[21]. To achieve a reasonable outcome from auctions, secondary operators must be strategic about their bids especially when there are multiple concurrent auctions offering similar radio resource characteristics.

However, given the discussion above, where billions of devices must communicate wirelessly in the existence of millions more base stations managed by many incumbent spectrum holders, the challenge for an operator is to select auctions that optimally deliver the QoS requirement and consequently increase the winning probability. Although there are several studies that discuss auctions in the context of dynamic spectrum allocation, most of these studies consider the problem of optimal bidding within one single auction. This paper differs from previous research that it takes into account several other auctions running concurrently. This assumption raises a number of problems, which to the best of our knowledge, have not been studied earlier. In this paper, we define and propose a solution to each problem separetely.

The remainder of the paper is as follows. In Section II, we introduce the system model. In Section III we present the problem formulations. We give the numerical results in Section IV. Finally, we conclude and discuss future work in Section $\mathrm{V}$.

\section{SySTEM MOdEL}

We consider a secondary spectrum market with multiple primary network operators (PNOs) and SNOs for idle spectrum in multiple trade windows. The idle spectrum usage rights are handed over to one SNO temporarily, following 


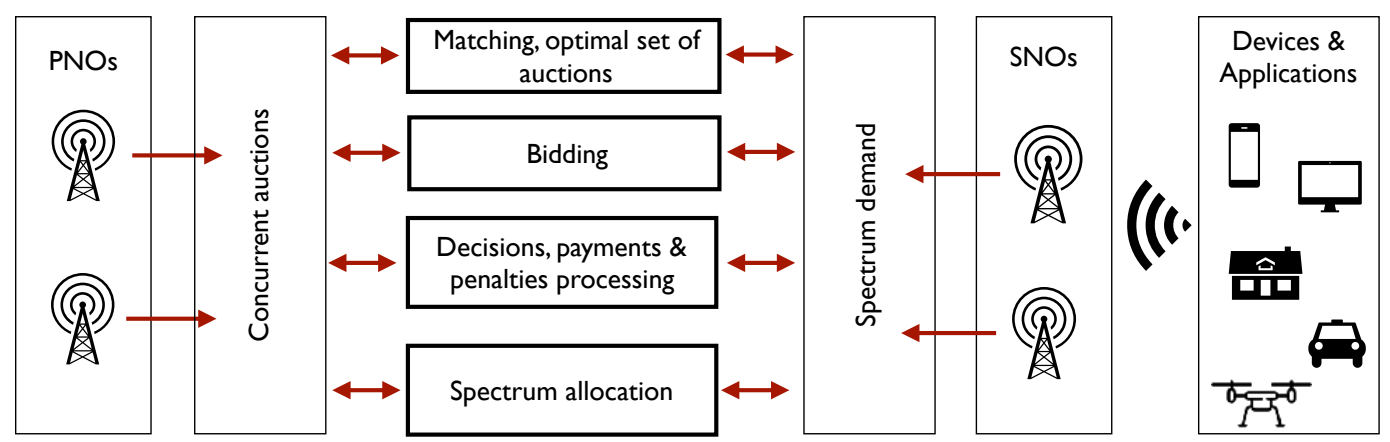

Fig. 1: Proposed system model

an auction, where multiple SNOs compete to acquire bandwidth resources. We denote $\mathcal{N}=\{1,2, \ldots, i, \ldots, N\}$ and $\mathcal{M}=\{1,2, \ldots, k, \ldots, M\}$ as the set of PNOs and SNOs, respectively, which operate in an area $\mathcal{R}$. The SNOs are actively seeking to borrow additional spectrum, say $\mathcal{D}=$ $\left\{d_{1}, d_{2}, \ldots, d_{k}, \ldots, d_{M}\right\}$, to support their users. Let

$$
\begin{aligned}
& \mathcal{S}=\left\{\left(a_{11}, a_{12}, \ldots, a_{1 L_{1}}\right),\left(a_{21}, a_{22}, \ldots, a_{2 L_{2}}\right),\right. \\
& \left.\ldots,\left(a_{N 1}, a_{N 2}, \ldots, a_{N L_{N}}\right)\right\}
\end{aligned}
$$

be the set of concurrent auctions, which run for a time duration $\left[t_{0}, t_{c}\right]$ where $L_{i}$ is the number of auctions of the $i$ th PNO. Each SNO has a desired bandwidth to borrow, which is in the narrow range of $\left[d_{k}, d_{k} \pm \delta_{k}\right], \delta_{k} \ll d_{k}$, where $d_{k}$ is the ideal matching and $\delta_{k}$ is the tolerance factor. Now let us make the following assumptions for modelling purposes:

- Opening and closing time of auctions are concurrent and SNOs make bids simultaneously.

- The quality of the auctioned spectrum blocks are identical but with varied transmit power constraints.

- In symmetric environments, when the valuation distribution is irregular, introducing asymmetric reserve prices can increase the PNO's revenue compared to an optimal common reserve price. Therefore we propose a scenario where multiple SNOs have multiple choices of auctions to enter their bids and where the reserve prices at these auctions vary.

Based on the system and assumptions described above, SNOs first select sets of closely matched feasible bids and reduce these sets further, for which the expected cost is lower and does not exceed a defined threshold. This is followed by the Vickrey auction mechanism, where the SNO wins a set of bids. The SNO then decides to select the bid with the total highest utility (utility surplus) and pull out from other bids by paying a predefined penalty costs.

\section{Problem Formulation AND SOlution}

In this section, we described the steps of matching and optimizations, which precede placing bids. The auction procedure, which describes a class of games with incomplete information, is addressed later in the paper [22].

\section{A. Matching procedure}

In a dynamic market, SNOs seek to borrow spectrum resources from PNOs by entering auctions. This, however, would raise the question of which auction an SNO should be assigned to. To formulate the problem, we assume that there are $\sum_{i=1}^{N} L_{i}$ auctions running and $M$ SNOs are bidding for a particular duration of time $\left[t_{0}, t_{c}\right]$. However, not all auctions are suitable for an SNO for duration of time $t$. Therefore, a bidder (SNO) will find the closely matched bids $\mathcal{S}^{\prime} \subseteq \mathcal{S}$. Close matching, measured in distance, is defined as the difference in a number of subcarriers available and required, considering the transmit power. Formally, the minimal distance solution to the above minimization problem can be stated as

$$
\left(\mathcal{P}_{1}\right): \min _{k} \sum_{i=1}^{N} \sum_{j=1}^{L}\left|\left(q_{a_{i j}}\left(W_{i}\right)-d_{k}\left(W_{i}\right)\right)\right| x_{i j k}
$$

s.t.

$$
\begin{aligned}
q_{a_{i j k}} x_{i j k} & \leq d_{k} \pm \delta_{k} \forall i, j, k \\
W_{i} & \leq \psi_{k} \forall i, k
\end{aligned}
$$

where $a_{i j}$ is the $j$ th auction of the $i$ th PNO; $x_{i j}$ is the binary $(\{0,1\})$ decision variables to decide whether to bid or not; $q_{a_{i j k}}$ is the number of subcarrier of the $a_{i j}$ th auction; $W_{i} \in$ $\mathbb{C}^{T}, \forall i$, is the complex weight vector transmitted from all users of a SNO and the constraint (4) represents that the total power is upper bounded by $\psi_{k}, \forall k \in \mathcal{M}$.

In problem $\left(\mathcal{P}_{1}\right)$, the minimization is performed over all combinations $\left(\mathcal{N} \otimes \mathcal{M} \otimes_{i=1}^{N} L_{i}\right)$. In this case, the optimizer assigns the SNOs with the highest number of subcarriers required to the auctions, satisfying constraint (3) and the power constraint $(4)$. The optimization problem $\left(\mathcal{P}_{1}\right)$ is a nonlinear mixed integer programming problem, which is solved using the brute force algorithm that provides global optimum.

\section{B. Cost and utility-based optimal set of auctions}

Suppose an SNO is searching for an appropriate auction. Each auction provides the same number of resources the SNO is required. However, each auction has a different reserve price (i.e. a minimum asking price). In a decentralized market, the SNOs will not know the prices ahead for any given trade window and it must express their interests in order to 
be informed of the reserve prices of subcarriers. The SNO chooses the number of enquiries to make, however, each enquiry costs $c>0$. Once the minimum reserve prices are revealed, the SNO finds more suitable auction sets based on the quoted lowest minimum reserve price. The problem is now to find the optimal number of enquiries $n^{*}$ for an SNO, which minimizes the total cost (i.e. total cost $=$ expected purchase cost + enquiring cost). Let us assume that the minimum reserve price $p_{i j}$ for an enquiry from the $i$ th PNO and $j$ th auction is a random variable that follows a distribution with $\mathrm{CDF}$ $F\left(p_{i j}\right)=\mathbb{P}\left(P \leq p_{i j}\right)$. Therefore,

$$
\mathbb{P}\left(P>p_{i j}\right)=1-F\left(p_{i j}\right) .
$$

With $n$ independent enquiries,

$$
\mathbb{P}\left(P>p_{i j}\right)=\left[1-F\left(p_{i j}\right)\right]^{n}
$$

is the probability of minimum reserve price. Hence, the empirical cumulative distribution (ECDF) of the lowest minimum reserve price for $n$ independent enquiries is

$$
F_{n}\left(p_{i j}\right)=1-\left[1-F\left(p_{i j}\right)\right]^{n} .
$$

Now the expected total cost of an SNO to borrow a unit subcarrier is

$$
\begin{aligned}
\mathbb{E}\left[c_{i j}(1, n)\right] & =\int_{\alpha_{1}}^{\alpha_{2}} p_{i j} \cdot \mathrm{d} F_{n}\left(p_{i j}\right) \\
& =\int_{\alpha_{1}}^{\alpha_{2}} p_{i j} \cdot f_{n}\left(p_{i j}\right) \mathrm{d} p_{i j} \\
& =\int_{\alpha_{1}}^{\alpha_{2}}\left[1-F\left(p_{i j}\right)\right]^{n} \mathrm{~d} p_{i j} .
\end{aligned}
$$

Therefore, the expected total cost to borrow $d_{k}$ subcarriers is

$$
\mathbb{E}\left[c_{i j}\left(d_{k}, n\right)\right]=d_{k} \cdot \int_{\alpha_{1}}^{\alpha_{2}}\left[1-F\left(p_{i j}\right)\right]^{n} \mathrm{~d} p_{i j},
$$

where $\alpha_{1}$ and $\alpha_{2}$ are the lowest and highest expected reserve price, respectively.

We now proceed to formulate the optimization problem $\left(\mathcal{P}_{2}\right)$ for $\mathcal{S}^{\prime \prime} \subseteq \mathcal{S}^{\prime}$.

$$
\left(\mathcal{P}_{2}\right): \quad \min _{n} \mathbb{E}\left[c_{i j}\left(d_{k}, n\right)\right]
$$

s.t.

$$
\begin{aligned}
c_{i j}+d_{k} p_{i j} & \leq \varepsilon_{k} \\
\alpha_{1} \leq p_{i j} & \leq \alpha_{2}
\end{aligned}
$$

where $\varepsilon_{k}$ is the total allowed expenditure for the $k$ th SNO. The solution to the above nonlinear mixed integer programming problem is obtained again by using the brute force algorithm and lies in the closed interval

$$
\begin{aligned}
\mathbb{E}\left[c_{i j}\left(d_{k}, n^{*}\right)-c_{i j}\right. & \left.\left(d_{k}, n^{*}+1\right)\right] \leq c_{i j} \\
\leq & \mathbb{E}\left[c_{i j}\left(d_{k}, n^{*}-1\right)-c\left(d_{k}, n^{*}\right)\right] .
\end{aligned}
$$

If the price dispersion is low $\left(\alpha_{2}-\alpha_{1} \approx 0\right)$ then solving the above problem $\left(\mathcal{P}_{2}\right)$ becomes trivial, however for significant price dispersion $\left(\alpha_{2} \gg \alpha_{1}\right)$, solving this problem could yield large savings and indeed good return of investment. A similar logic can be applied for $d_{k}$, where as spectrum demand is high, the SNOs find it more efficient to solve $\mathcal{P}_{2}$.

\section{Vickery strategy with aborting options: selecting the final set of auctions}

In each auction, there are $M$ potential SNOs bidding for the spectrum, each of whom has a privately known value for the auctioned radio resources. Each potential SNO views the private value of competitors as a random variable $V$, which represents an independent draw from the cumulative distribution function $(\mathrm{CDF}) F_{k}(v)$ that is twice continuously differentiable and has a strictly positive probability density function (PDF) $f_{k}(v)$ on a compact support $\left[v_{\min }, v_{\max }\right]$. To reduce clutter, let $Z$ denote $V_{(1: M-1)}$, the highest valuation of $(M-1)$ bids from the $F_{k}(v)$; in symbols, $Z=\max \left\{V_{1}, V_{2}, \ldots, V_{(M-1)}\right\}$. The random variable $Z$ represents the highest of a bidder's $(M-1)$ opponents at the auction. Given that valuations are distributed independently and identically, the CDF and PDF of $Z$ are $F_{Z}(z)=F_{k}(z)^{M-1}$ and $f_{Z}(z)=(M-1) F_{V}(z)^{M-2} f_{k}(z)$, respectively.

The valuation of the $k$ th SNO of the radio resources is independently and identically distributed according to the CDF $F_{k}(\cdot)$ on the interval $\left[d_{k} p_{i j}, \varepsilon_{k}\right]$. According to the proposed auction mechanism in Algorithm 1 each SNO submits a bid with price

$$
Y_{k} \in\left[d_{k} p_{i j}, \varepsilon_{k}\right), d_{k} p_{i j}>0
$$

All bids $\left[d_{k} p_{i j}, \varepsilon_{k}\right)$ are highly competitive. An SNO submitting the highest bid wins the auction and makes a payment equal to the second highest bid $Y_{k}^{(b-1)}$ or the reserved price $d_{k} p_{i j}$ in the case, where there is only one submitted bid. In the case, where two or more SNOs submit the same bid and it turns out to be the highest, then the tie is resolved arbitrary with a uniform randomization and the winner pay the highest bid $Y_{k}^{(b)}$. Any bid $Y_{k}<d_{k} p_{i j}$ is a noncompetitive bid, equivalent to not participating in the auction. Most importantly, an SNO bidding $Y_{k}<d_{k} p_{i j}$ receives a payoff of zero, irrespective of the others' bids (and even if it is the only bid). The payoff of an SNO, who values the radio resource as $V_{k}$ and wins the auction with bid $Y_{k}$ is $V_{k}-Y_{k}^{(b-1)}$. In this case, the unique Bayesian-Nash equilibrium, where all bidders adopt the bidding strategy is

$$
\beta_{V_{k}}= \begin{cases}Y_{k}, & \text { if } V_{k}<d_{k} p_{i j} \\ V_{k}-\int_{d_{k} p_{i j}}^{V_{k}}\left[\frac{F(z)}{F\left(V_{k}\right)}\right]^{[M-1]} d z, & \text { if } V_{k} \geq d_{k} p_{i j}\end{cases}
$$

where $F\left(V_{k}\right)$ is the CDF of the valuation of the $k$ th SNO. The assumption that the SNOs may place multiple bids in multiple auctions to increase their chances of gaining spectrum access raises the need to consider aborted bids and hence the second 
case of equation (14) can be written as

$$
\beta_{V_{k}}=V_{k}-\int_{d_{k} p_{i j}}^{V_{k}}\left[\frac{F(z)}{F\left(V_{k}\right)}\right]^{\left[M-M^{\prime}-1\right]} d z, \text { if } V_{k} \geq d_{k} p_{i j}
$$

where $M^{\prime}$ is the number of aborted SNOs from the auction $i j$.

\section{Selection of the best auction}

Consider an SNO $k \in \mathcal{M}$, which is distinguished by features divided into two vectors $\sigma$ and $\lambda$. PNO provides the service to the $k$ th SNO after winning an auction. The vector $\sigma$ describes any SNO features that affect the cost of the PNO. The vector $\lambda$ contains all other SNOs features relevant to its requirements and constraints, such as number of channels and cost.

The auctions available $\mathcal{S}^{\prime \prime}$ are described by reserve prices $p$ and plan features $\phi$, where $\phi$ includes taxes, types of penalty and so on. Uncertain information of the future characteristics (e.g. traffic, leasing agreement) and strategies of the network (e.g. security, regulation) in various states $s \in \mathbb{S}$, where the probability distribution over the strategy of the $k$ th SNO is $G_{k}=G\left(s \mid \sigma_{k}\right)$. The expected utility of winning an auction $\left(p_{i j}, \phi_{i j}\right)$ to a $\operatorname{SNO}\left(\lambda_{k}, \sigma_{k}\right)$ is

$$
v\left(p_{i j}, \phi_{i j}, \lambda_{k}, \sigma_{k}\right)=\int u\left(s, p_{i j}, \phi_{i j}, \lambda_{k}, \sigma_{k}\right) \cdot G\left(s \mid \sigma_{k}\right) \mathrm{d} s .
$$

With the expected utility defined as above, an SNO chooses to bid in an auction that generate the highest expected utility

$$
v\left(p_{i j}, \phi_{i j}, \lambda_{k}, \sigma_{k}\right) \geq v\left(p_{l m}, \phi_{l m}, \lambda_{k}, \sigma_{k}\right) \forall l, m \in \mathcal{S}^{\prime \prime} .
$$

There are also costs to the PNOs due to refund to the SNO in case the SNO will not get the full expected services. Suppose $c\left(\phi_{i j}, \lambda_{k}\right)$ is expected cost to the $i$ th PNO providing the $j$ th auction

$$
c\left(\phi_{i j}, \lambda_{k}\right)=\int \eta\left(s, \phi_{i j}, \sigma_{k}\right) \cdot G\left(s \mid \sigma_{k}\right) \mathrm{d} s .
$$

where $\eta$ is the state-specific cost of the $i$ th PNO providing the $j$ th auction. The expected cost of the PNO depends on the SNO that determines the distribution $G\left(s \mid \sigma_{k}\right)$, but not on $\sigma_{k}$. Adding the interference parameter $\delta_{k}$, we now define the total utility surplus (TUS) as

$$
W=\sum_{k \in \mathcal{M}} \sum_{i j \in \mathcal{S}^{\prime \prime}} I\left(k_{i j}\right) \cdot\left[v\left(p_{i j}, \phi_{i j}, \lambda_{k}, \sigma_{k}\right)-c\left(\phi_{i j}, \lambda_{k}\right)\right] \text {, }
$$

where $I\left(k_{i j}\right)$ is an indicator function for the set of auctions bid by the SNO $k$. After winning a set of bids, the choice of bids can further be reduced by maximizing utility discussed above. We now want to obtain $W$ which is the standard unconstrained efficiency condition for each SNO by sorting auctions according to their TUS value.
E. Auction design under account balance and penalty with optimal sets

\section{Algorithm 1: Vickrey auction algorithm with refund mechanism}

1 Initialization: Let $\mathbf{a}=\left\{\mathbf{a}_{1}, \mathbf{a}_{2}, \ldots, \mathbf{a}_{N}\right\}$ set of auctions for $N$ PNOs; Number of bidders $1,2, \ldots, M$; Starting and closing auction time $\left[t_{0}, t_{c}\right]$; Minimum price matrix $\mathbf{P}_{l}, Y_{k}^{(b)} \leftarrow$ best price; $\mathbf{P}_{l}, Y_{k}^{(b-1)} \leftarrow$ second best price; $Y_{k} \leftarrow$ value of the $j$ th bid

2 while $t_{c} \neq 0$ do

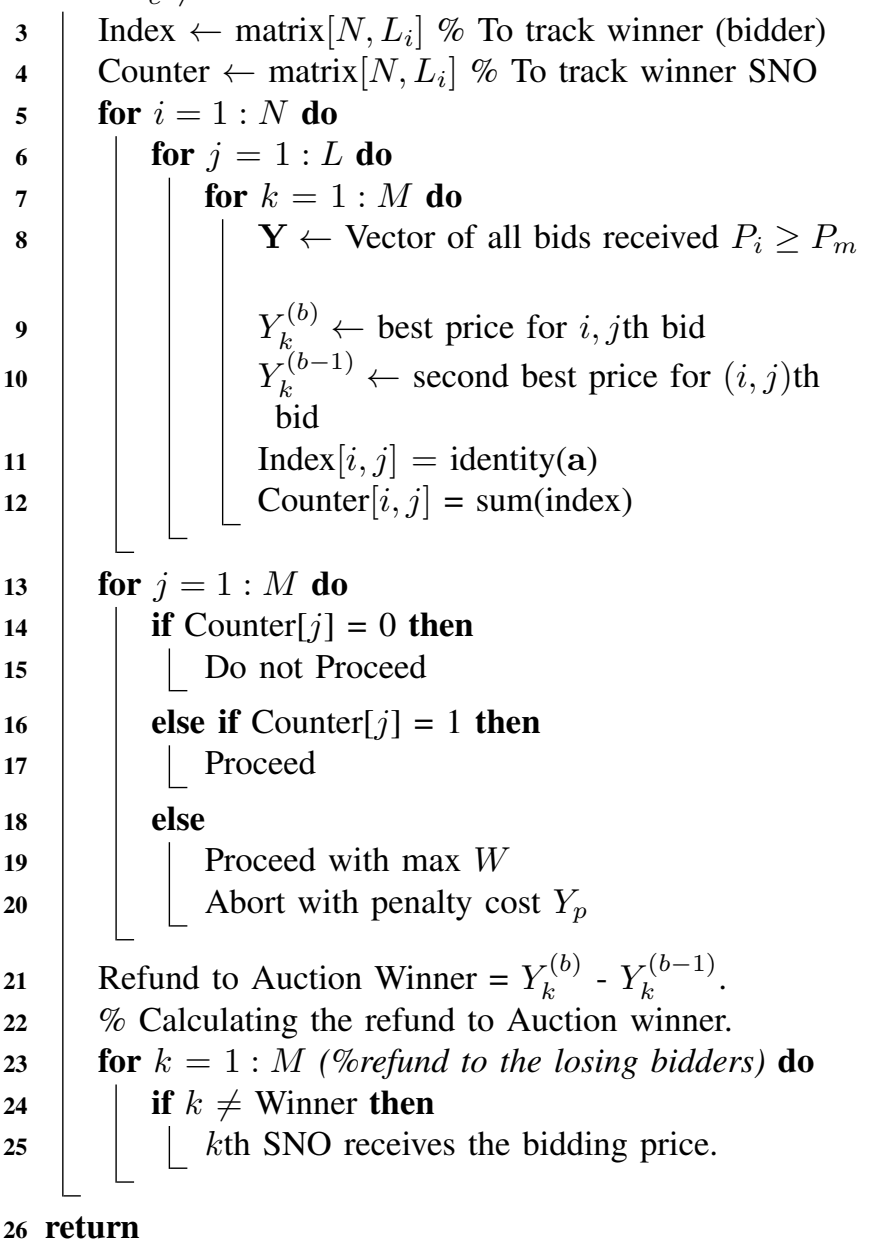

There are penalties involved for pulling out after winning an auction. The winner, which decides to abort after winning, pays an initial fixed penalty in form of monetary value plus additional amount based on the volume of resources in the bid. In the simplest case, it can be written mathematically as

$$
Y_{p}=c_{0}+\sum_{i=1}^{p-1} c_{i} x_{i}
$$

where $p(\leq n)$ is the number of bids won, $c_{0}$ is the fixed penalty, $c_{i}$ is the variable penalty per unit and $x_{i}$ is the amount of bid resources. The mathematical form can also have the 
following exponential form

$$
Y_{p}=c_{0} \exp \left(\sum_{i=1}^{p-1} c_{i} x_{i}\right) .
$$

Once an auction is complete, the winner is allowed to use the spectrum for the specified time and within the area boundaries. This approach simplifies the mechanisms within the spectrum market, allowing exchange of radio resources between operators (or between users and operators with some adjustments) and facilitating transactions. The following steps summarize the methods involved in the spectrum auction management under oligopoly market structure

1) select a feasible set of bids $\mathcal{S}^{\prime} \subseteq \mathcal{S}$, using $\left(\mathcal{P}_{1}\right)$

2) select the number of the optimal auctions $n^{*}$ using $\left(\mathcal{P}_{2}\right)$

3) run Algorithm 1 to identify auctions' winners and losers; the outcome of bidding in $n^{*}$ auctions: win $\rightarrow 0$, win $\rightarrow 1$ or win $\rightarrow p, p \leq n$

4) if an SNO loses all auctions (0), do not proceed and the SNO obtains all his bidding payments as a form of refund

5) if an SNO wins (1), proceed by allocating the subcarriers to the SNO

6) if an $\mathrm{SNO}$ wins $(p)$; run $\left(\mathcal{P}_{3}\right)$ to sort bids based on maximum utility (utility surplus); abort from $(p-1)$ keeping the auction won with highest utility $(\mathcal{W})$.

7) proceed by allocating the subcarriers to the SNO

\section{NumericAl RESUlts}

In this section, we show the results of our numerical study, which validate and verify our proposed model and algorithm. We will discuss the performances of the model formulation of bidding in auctions under various parameter settings.

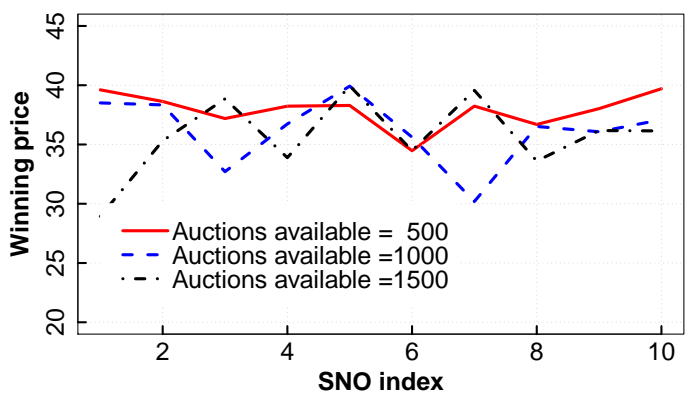

Fig. 2: SNOs winning prices when bid for all auctions

One of the main goals of the model formulation and proposed algorithm is to choose the optimal bid and increase the probability of winning while satisfying some constraints. It is customary to show that heuristic method cannot guarantee the higher probability of winning with increased cost when bidding all auctions. Figure 2 shows that the cost of the SNOs remains, on an average, constant when a bid is made for all auctions. Therefore, it is interesting to see how the optimal choice of the proposed method reduces cost while ensuring the chosen set of auctions are most suitable (ensured via the constraints).

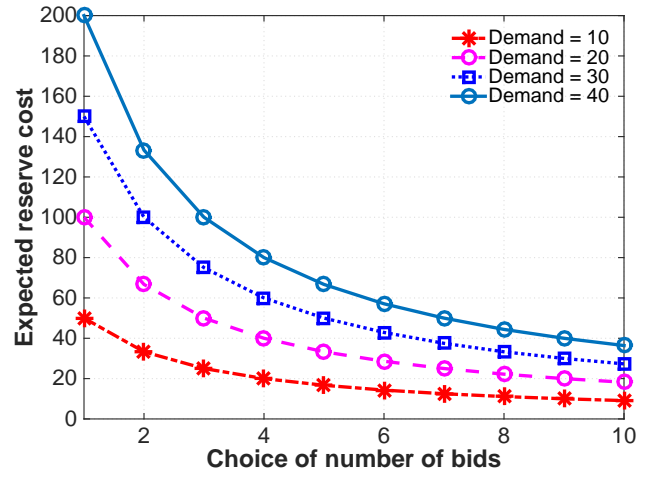

Fig. 3: Expected reserve costs of bidding vs number of bids of an SNO

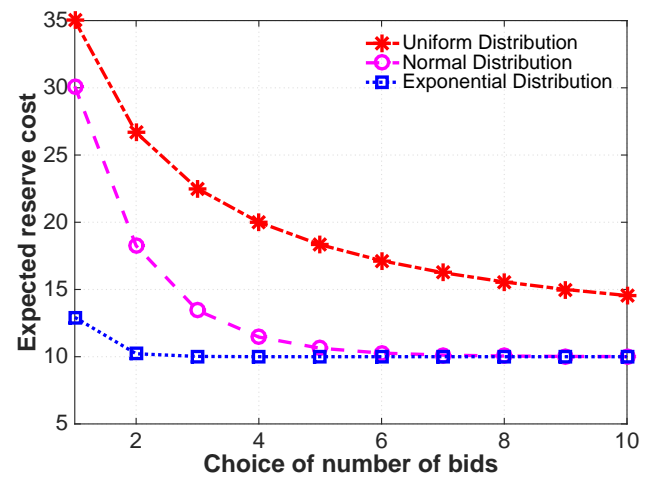

Fig. 4: Expected reserve cost for various assumed empirical distributions

The PNOs disclose the number of available subcarriers to the SNOs while the reserve prices for each auction remain concealed. The SNOs, upon matching optimization algorithm, determine the number and auctions to request reserve prices. This allows the SNOs to reduce its level of uncertainty as well as reduce the processing time of requests (bids). In Figure 3, we show that for price dispersion in the range between 10 and

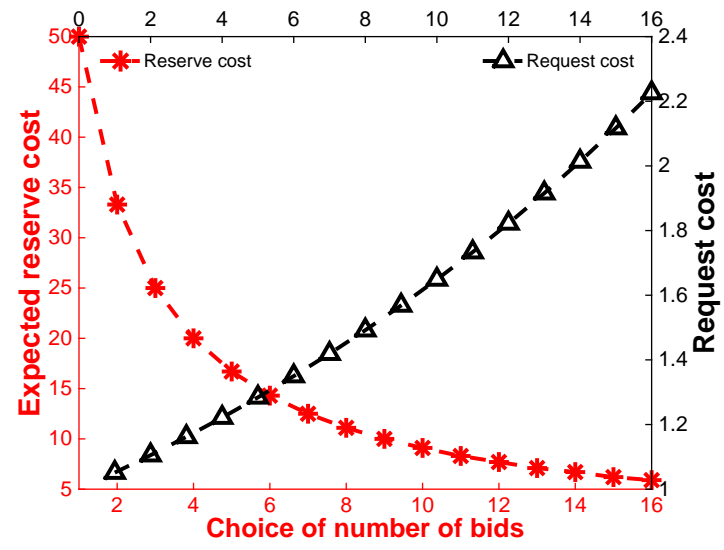

Fig. 5: Expected reserve cost and bid request cost vs number of bids of a SNO 
50 , the SNO is able to reduce its costs by requesting prices from more auctions, however, we can see from the figure that at some point on the curve, the effect of increasing the number of requests does not change the costs of the SNO. Finding the optimal point $\left(n^{*}\right)$ at which the costs are not reduced is achieved by following the second-order condition which is presented in equation (13).

As expected, the average reserve cost decreases with higher number bids since more bidding options are available with the higher number of bids, which allows bidding the cheaper ones (Figure 3 and 4). Figure 4 show sensitivity of the average reserve cost for various assumed empirical distributions (uniform, exponential and Gaussian). On the contrary, the higher number of biddings incur higher bid request costs. Therefore, an optimal solution is required, which will allow an SNO (bidder) to choose an optimal number of bids with a fixed amount spending on 'requests' to reveal bidding costs. Simulation experiments reveal that the expected reserve cost is an exponential decay function (decreasing) while the bid request cost is an exponential function (increasing). The equilibrium state is the point where both lines cross. With the set of chosen parameters, our simulation results show that the optimal number of bids is six (Figure 5), which is further reduced by using the total utility surplus function.

\section{CONCLUSiON}

Our main contribution in the paper is that we formulated a spectrum sharing problem under the competition of multiple SNOs while a number of primary operators are willing to lease their secondary radio resources through multiple concurrent auctions. We found that the choice for the secondary spectrum purchase is vast and requires the solution of a number of smaller optimization problems, for instance, choice of the appropriate amount of spectrum available from a number of sellers (PNOs), number of auctions to bid, auction mechanism, utility-based choice of an auction.

Our proposed approach is a general scheme that obtains the optimal bids and wins a set of most feasible bids from a set of auctions while minimizing cost and maximizing utility. The time complexity of the entire algorithm is polynomial. We also evaluated the performance of the proposed algorithm via numerical studies with various number of auctions from a set of PNOs. Numerical results provide insight into the selection of maximum matched, choice of the optimal number of bids and the maximal utility bid selection under different spectrum demands. In our future work, we plan to extend our unified framework of secondary resource allocation through auctions among the SNOs with consideration to the sensitivity of the constraints, such as, latency, security, capacity, throughput and scalability.

\section{REFERENCES}

[1] A. Osseiran, F. Boccardi, V. Braun, K. Kusume, P. Marsch, M. Maternia, O. Queseth, M. Schellmann, H. Schotten, H. Taoka et al., "Scenarios for $5 \mathrm{G}$ mobile and wireless communications: the vision of the metis project," IEEE Communications Magazine, vol. 52, no. 5, pp. 26-35, 2014.
[2] G. P. Fettweis, "The tactile internet: Applications and challenges," IEEE Vehicular Technology Magazine, vol. 9, no. 1, pp. 64-70, 2014.

[3] V. Petrov, M. A. Lema, M. Gapeyenko, K. Antonakoglou, D. Moltchanov, F. Sardis, A. Samuylov, S. Andreev, Y. Koucheryavy, and M. Dohler, "Achieving end-to-end reliability of mission-critical traffic in softwarized 5G networks," IEEE Journal on Selected Areas in Communications, vol. PP, no. 99, pp. 1-1, 2018.

[4] M. R. Palattella, M. Dohler, A. Grieco, G. Rizzo, J. Torsner, T. Engel, and L. Ladid, "Internet of things in the 5G era: Enablers, architecture, and business models," IEEE Journal on Selected Areas in Communications, vol. 34, no. 3, pp. 510-527, 2016.

[5] T. W. Hazlett, "A law \& (and) economics approach to spectrum property rights: A response to weiser and hatfield," Geo. Mason L. Rev., vol. 15, p. 975, 2007.

[6] S. Buzzi, I. Chih-Lin, T. E. Klein, H. V. Poor, C. Yang, and A. Zappone, "A survey of energy-efficient techniques for $5 \mathrm{G}$ networks and challenges ahead," IEEE Journal on Selected Areas in Communications, vol. 34, no. 4, pp. 697-709, 2016.

[7] M. Hawa, A. AlAmmouri, A. Alhiary, and N. Alhamad, "Distributed opportunistic spectrum sharing in cognitive radio networks," international journal of communication systems, vol. 30, no. 7, 2017.

[8] M. Mirahsan, R. Schoenen, and H. Yanikomeroglu, "Hethetnets: Heterogeneous traffic distribution in heterogeneous wireless cellular networks," IEEE Journal on Selected Areas in Communications, vol. 33, no. 10, pp. 2252-2265, Oct 2015.

[9] M. M. Buddhikot, I. Kennedy, F. Mullany, and H. Viswanathan, "Ultrabroadband femtocells via opportunistic reuse of multi-operator and multi-service spectrum," Bell Labs Technical Journal, vol. 13, no. 4, pp. 129-143, 2009.

[10] I. Sugathapala, I. Kovacevic, B. Lorenzo, S. Glisic et al., "Quantifying benefits in a business portfolio for multi-operator spectrum sharing," IEEE Transactions on Wireless Communications, vol. 14, no. 12, pp. 6635-6649, 2015.

[11] R. Abozariba, M. Asaduzzaman, and M. Patwary, "Radio resource sharing framework for cooperative multioperator networks with dynamic overflow modeling," IEEE Transactions on Vehicular Technology, vol. 66, no. 3, pp. 2433-2447, 2017.

[12] M. Asaduzzaman, R. Abozariba, and M. Patwary, "Dynamic spectrum sharing optimization and post-optimization analysis with multiple operators in cellular networks," IEEE Transactions on Wireless Communications, vol. 17, no. 3, pp. 1589-1603, 2018.

[13] A. Chandra, M. Sammour, and J. Wang, "Dynamic resource allocation, scheduling and signaling for variable data rate service in LTE," Jan. 18 2018, uS Patent App. 15/678,934.

[14] C. S. Hyder, T. D. Jeitschko, and L. Xiao, "Bid and time truthful online auctions in dynamic spectrum markets," IEEE Transactions on Cognitive Communications and Networking, vol. 3, no. 1, pp. 82-96, 2017.

[15] Z. Liu and C. Li, "On spectrum allocation in cognitive radio networks: a double auction-based methodology," Wireless Networks, vol. 23, no. 2, pp. 453-466, 2017.

[16] S. Gandhi, C. Buragohain, L. Cao, H. Zheng, and S. Suri, "A general framework for wireless spectrum auctions," in New Frontiers in Dynamic Spectrum Access Networks, 2007. DySPAN 2007. 2nd IEEE International Symposium on. IEEE, 2007, pp. 22-33.

[17] X. Chen, L. Xing, T. Qiu, and Z. Li, "An auction-based spectrum leasing mechanism for mobile macro-femtocell networks of IoT," Sensors, vol. 17, no. 2, p. 380, 2017.

[18] O. Agmon Ben-Yehuda, M. Ben-Yehuda, A. Schuster, and D. Tsafrir, "Deconstructing Amazon EC2 spot instance pricing," ACM Transactions on Economics and Computation, vol. 1, no. 3, p. 16, 2013.

[19] M. A. Islam, X. Ren, S. Ren, A. Wierman, and X. Wang, "A market approach for handling power emergencies in multi-tenant data center," in High Performance Computer Architecture (HPCA), 2016 IEEE International Symposium on. IEEE, 2016, pp. 432-443.

[20] F. Teng and F. Magoules, "Resource pricing and equilibrium allocation policy in cloud computing," in Computer and information technology (CIT), 2010 IEEE 10th international conference on. IEEE, 2010, pp. 195-202.

[21] J. N. Tsitsiklis and Y. Xu, "Efficiency loss in a cournot oligopoly with convex market demand," Journal of Mathematical Economics, vol. 53, pp. 46-58, 2014.

[22] J. C. Harsanyi, "Games with incomplete information played by bayesian players, i-iii part i. the basic model," Management science, vol. 14, no. 3, pp. 159-182, 1967. 